\title{
Discriminatiing Between Degum Distribution and Burr-III Distribution
}

\author{
B. Srinivasa Rao ${ }^{1}$, P. Sricharani ${ }^{2 *}$ \\ ${ }^{1}$ Department of Mathematics and Humanities, R.V.R \& J.C College of Engineering, Guntur, India \\ ${ }^{2}$ Department of Basic science, Shri Vishnu Engineering College for Women(Autonomous), Bhimavaram, India \\ *Corresponding author: charani.ravi657@gmail.com
}

Available online at: www.isroset.org

Received: 12/Sept/2018, Accepted: 03/Oct/2018, Online: 31/Oct/2018

\begin{abstract}
Test statistics based on Likelihood function, population Quantiles are suggested to discriminate between Dagum distribution and Burr-III distribution. Because of the non tractability of their exact sampling distributions, the percentiles of the proposed test statistics are tabulated with the help of simulated sampling distributions of the test statistics. The power of the test statistics are also tabulated and a comparative study w.r.t the powers for a given sample and the level of significance are worked out.
\end{abstract}

Key words and phrases- Dagum distribution(DD), Burr-III distribution(BD), Likelihood function, Quantiles, power of the test.

\section{INTRODUCTION}

Having experienced with the lack of analytical expression for the classical maximum likelihood estimation (MLE) of parameters in Dagum distribution, we propose to study whether any other standard model be an alternative to Dagum distribution with a reasonably admissible risk. Accordingly, we have chosen Burr type III model to test whether it can be an alternative to Dagum distribution. This aspect is viewed as the problem of discriminating between Dagum and Burr III models where Dagum is null population. We have considered the principle of Likelihood ratio criterion in a practically usable way. Hence we may call our procedure as likelihood ratio type procedure. The distinction is- in the likelihood procedure to the classical MLE is used for both null and alternative populations. In the likelihood ratio type procedure we use any admissible estimators of concerned models as given by $[1,2,3]$.

The Probability density function (pdf) of Dagum distribution is given by

$$
f(x, b, a, p)=\frac{a p}{x}\left(\frac{\left(\frac{x}{b}\right)^{a p}}{\left(\left(\frac{x}{b}\right)^{a}+1\right)^{p+1}}, x>0, a>0, b>0, p>0 .\right.
$$

Its cumulative distribution function (cdf) is

$$
F(x, b, a, p)=\left(1+\left(\frac{x}{b}\right)^{-a}\right)^{-p}, x>0, a>0, b>0, p>0 .
$$

The likelihood function of Dagum distribution is

$$
L(x, a, p, b)=\frac{(a p)^{n}\left(\prod_{i=1}^{n} x_{i}^{(a p-1)}\right)}{(b)^{n a p}\left(\prod_{i=1}^{n}\left(1+\left(\frac{x_{i}}{b}\right)^{a}\right)^{p+1}\right)}
$$

The Dagum distribution is a skewed, uni-modal distribution on the positive real line.

The probability density function (pdf) of Burr-III distribution is given by

$$
f(x ; c, k)=c k \frac{x^{-(c+1)}}{\left(1+x^{-c}\right)^{k+1}}
$$

The cumulative distribution function(cdf) is

$$
F(x ; c, k)=\left(1+x^{-c}\right)^{-k}
$$

The likelihood function of Burr-III distribution is

$$
L(x, c, k)=(c k)^{n}\left(\prod_{i=1}^{n}\left(x_{i}^{-(c+1)}\right)\left(1+\left(x_{i}\right)^{-c}\right)^{-(k+1)}\right)
$$


Many people have studied the importance of these two distributions as life testing models. The related works include [4\&5]. In fact inferential properties of Dagum distribution are simple, efficient and mathematically tractable, where as it is not the same case with Burr-III distribution. We are therefore motivated to study whether Dagum distribution is a reasonable alternative to Bur-III distribution at least for the sake of adopting the analytical, powerful inferential characteristics of the data following Burr-III distribution. With this backdrop we suggested two different test statistics to discriminate Dagum distribution(with $a=0.5, p=0.5$ and $b=2 / 3$ ) and Burr-III distribution(with $\mathrm{c}=0.5$ and $\mathrm{k}=0.5$ ). The pdfs and cdfs plotted for the mentioned values of both the distributions are given below
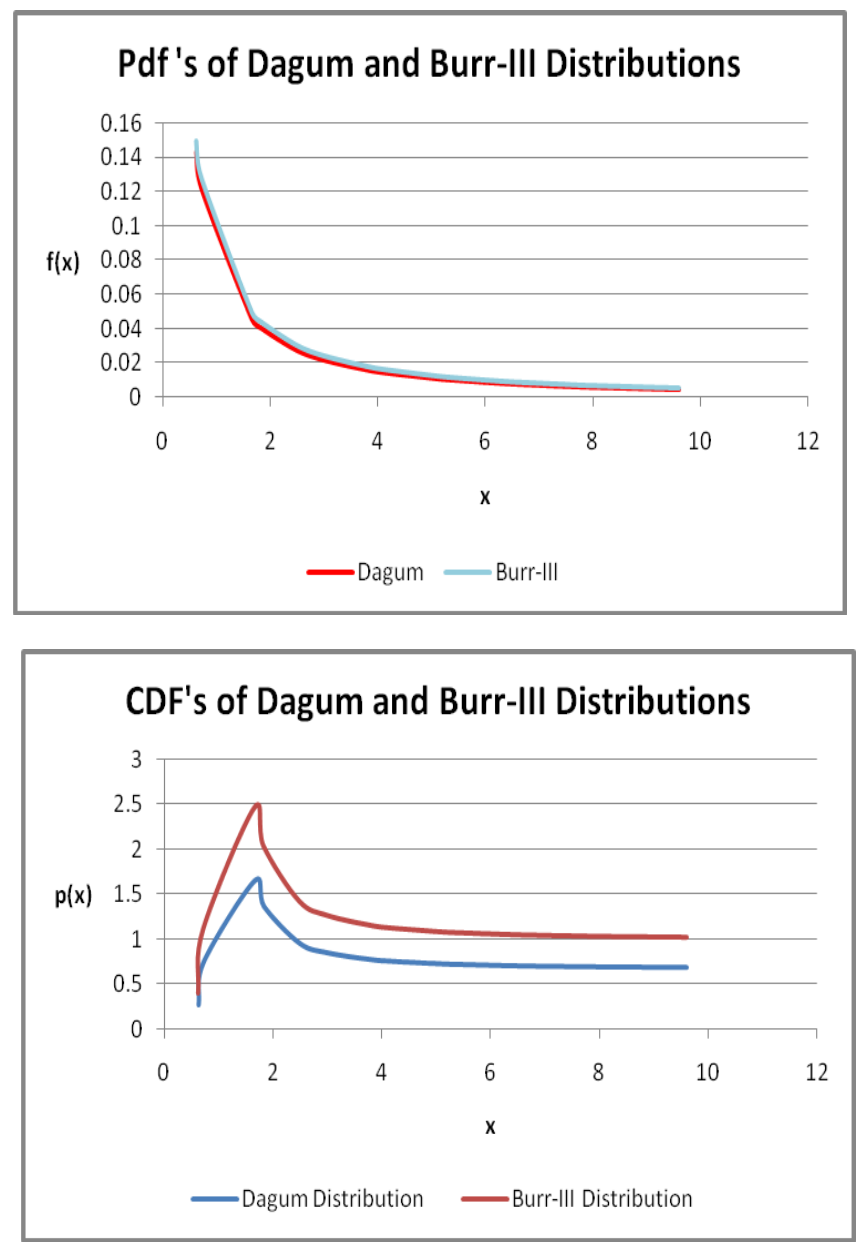

The basic distribution characteristics of Dagum distribution(DD) and its Properties are Presented in Section I. The rest of the paper is organized as follows. The proposed test statistics based on ratio of likelihood functions along with their percentiles and power values are explained in section II and Population quantiles explained in section III along with their percentiles and power values. Summary and Conclusions are given in section IV.

\section{II .TEST STATISTIC BASED ON LIKELIHOOD RATIO}

Let us consider Dagum distribution as the null population say $\left(P_{0}\right)$, the Bur-III distribution is considered as the alternative population say $\left(P_{1}\right)$. If a random sample of $x_{1}, x_{2}, \ldots x_{n}$ is drawn from $P_{0}$ evaluate the estimation of

the parameters of $P_{0}$ using the given sample of $x_{1}, x_{2}, \ldots x_{n}$.

Let $\hat{P}_{0}$ denote the value of the likelihood function of the sample $x_{1}, x_{2}, \ldots x_{n}$ with respect to null population, at the estimates of its parameters using $x_{1}, x_{2}, \ldots x_{n}$. Let $\hat{P}_{1}$ denote the value of likelihood function w.r.t to alternative population, the estimates of $P_{1}$ using the sample $x_{1}, x_{2}, \ldots x_{n}$. The ratio $\frac{L_{1}}{L_{0}}$ in a way represents the ratio of the likelihood of $P_{1}$ to that of $P_{0}$ with a sample drawn from $P_{0}$. Therefore $\frac{P_{1}}{P_{0}}$ is the ratio of a smallest probability to a larger probability and hence is expected to be small. The null hypothesis "H0: The sample is drawn from $P_{0}$ " can be tested using the percentiles of the likelihood ratio $\frac{L_{1}}{L_{0}}$. Therefore statistics $T_{1}=\frac{L_{1}}{L_{0}}$ can be taken as test statistics to test the above null hypothesis.

Since the distribution of $\frac{L_{1}}{L_{0}}$ is not analytical, we have computed the percentiles of empirical sampling distributions of $T_{1}=\frac{L_{1}}{L_{0}}$ with the help of 10,000 simulation runs of sample size $n=2, . ., 10$ are given in table 1 . 
Table1: Percentiles of Sampling Distribution of $T_{1}=\frac{L_{1}}{L_{0}}$

\begin{tabular}{|c|c|c|c|c|c|c|c|c|c|}
\hline $\mathrm{n}$ & $\mathbf{0 . 9 9 8 6 5}$ & $\mathbf{0 . 9 9}$ & $\mathbf{0 . 9 7 5}$ & $\mathbf{0 . 9 5}$ & $\mathbf{0 . 0 5}$ & $\mathbf{0 . 0 2 5}$ & $\mathbf{0 . 0 1}$ & $\mathbf{0 . 0 0 5 0}$ & $\mathbf{0 . 0 0 1 3 5}$ \\
\hline 2 & 1.4956 & 1.4785 & 1.4538 & 1.3936 & 0.8368 & 0.8267 & 0.8204 & 0.8185 & 0.8171 \\
\hline 3 & 1.6160 & 1.4503 & 1.3655 & 1.2965 & 0.7835 & 0.7645 & 0.7522 & 0.7465 & 0.7411 \\
\hline 4 & 1.7305 & 1.5369 & 1.1317 & 1.3438 & 0.7479 & 0.7202 & 0.7002 & 0.6887 & 0.6790 \\
\hline 5 & 1.8640 & 1.6267 & 1.5076 & 1.3992 & 0.7179 & 0.6866 & 0.6602 & 0.6453 & 0.6259 \\
\hline 6 & 1.9104 & 1.6754 & 1.5384 & 1.4644 & 0.6974 & 0.6595 & 0.6239 & 0.6067 & 0.5914 \\
\hline 7 & 2.1733 & 1.7277 & 1.6005 & 1.4276 & 0.6734 & 0.6342 & 0.5956 & 0.5748 & 0.5453 \\
\hline 8 & 2.3034 & 1.8447 & 1.6608 & 1.5067 & 0.6490 & 0.6072 & 0.5682 & 0.5427 & 0.5157 \\
\hline 9 & 2.2789 & 1.8422 & 1.6568 & 1.5151 & 0.6340 & 0.5892 & 0.5473 & 0.5224 & 0.4849 \\
\hline 10 & 2.3018 & 1.9082 & 1.7136 & 1.5569 & 0.6150 & 0.5699 & 0.5216 & 0.4970 & 0.4584 \\
\hline
\end{tabular}

The power of the test statistic $\mathrm{T}_{1}$ is also tabulate for 3 different levels of significance $(1 \%, 2.5 \% \& 5 \%)$ at the sample sizes $n=2,3 \ldots 10$.by simulating sampling from $P_{1}$ and using the values of $T_{1}$. The count of $T_{1}$ values that fall beyond the table values of table -1 . These are given in the Table -2 .

Table 2: Power of $T_{1}$

\begin{tabular}{|c|c|c|c|}
\hline $\mathrm{n}$ & $\mathbf{0 . 9 9 0 0}$ & $\mathbf{0 . 9 7 5 0}$ & $\mathbf{0 . 9 5 0 0}$ \\
\hline 2 & 9997 & 9987 & 9919 \\
\hline 3 & 9846 & 9650 & 9328 \\
\hline 4 & 9831 & 9627 & 9300 \\
\hline 5 & 9885 & 9638 & 9282 \\
\hline 6 & 9822 & 9752 & 9240 \\
\hline 7 & 9789 & 9528 & 9142 \\
\hline 8 & 9826 & 9610 & 9245 \\
\hline 9 & 9788 & 9488 & 9107 \\
\hline 10 & 9771 & 9528 & 9100 \\
\hline
\end{tabular}

These tables indicate that even with the help of a small sample of size as small as 2 the power remains to be at more than $99 \%$. It is therefore concluded that the T1 statistic proposed in this section cannot discriminate between the null and alternative population with a high power values.

\section{TEST STATISTICS BASED ON QUANTILES}

Let $x_{1}, x_{2}, \ldots x_{n}$ denote sample from DD. The correlation type goodness of fit test as given by [6\&7] in this case using percentiles can be formed as

$$
T_{2}=\frac{\sum_{i=1}^{n} X_{i} v_{i}}{\sqrt{\left(\sum_{i=1}^{n} X_{i}^{2} \sum_{i=1}^{n} v_{i}^{2}\right)}}
$$

This statistic represent the correlation between $x_{i} \& v_{i}$ $\mathrm{i}=1,2 \ldots \mathrm{n}$ where $\mathrm{v}_{\mathrm{i}}$ is the $\mathrm{i}^{\text {th }}$ quantile of the null population. The statistic $T_{2}$ is simulated through Monte Carlo method based on 10,000 simulations. .Table- 3 represents the percentiles of $T_{2}$ for sample sizes $n=2,3 \ldots 10$ and various levels of significance

Table3: Percentiles Of T2 Based On Quantile

\begin{tabular}{|c|c|c|c|c|c|c|c|c|c|}
\hline $\mathrm{n}$ & $\mathbf{0 . 9 9 8 6 5}$ & $\mathbf{0 . 9 9 0 0}$ & $\mathbf{0 . 9 7 5 0}$ & $\mathbf{0 . 9 5 0 0}$ & $\mathbf{0 . 0 5 0 0}$ & $\mathbf{0 . 0 2 5 0}$ & $\mathbf{0 . 0 1}$ & $\mathbf{0 . 0 0 5}$ & $\mathbf{0 . 0 0 1 3 5}$ \\
\hline 2 & 1 & 0.9999 & 0.9999 & 0.9999 & 0.0244 & 0.0244 & 0.0244 & 0.0244 & 0.0244 \\
\hline 3 & 0.9999 & 0.9998 & 0.9992 & 0.9984 & 0.0032 & 0.0027 & 0.0026 & 0.0026 & 0.0026 \\
\hline 4 & 0.9998 & 0.9991 & 0.9972 & 0.9952 & 0.0017 & 0.0007 & 0.0005 & 0.0005 & 0.0005 \\
\hline 5 & 0.9996 & 0.9969 & 0.9936 & 0.9923 & 0.0020 & 0.0005 & 0.0002 & 0.0001 & 0.0002 \\
\hline 6 & 0.9989 & 0.9937 & 0.9901 & 0.9896 & 0.0012 & 0.0007 & 0.0001 & 0.0001 & 0.0001 \\
\hline 7 & 0.9987 & 0.9931 & 0.9883 & 0.9874 & 0.0025 & 0.0012 & 0.0005 & 0.0003 & 0.0001 \\
\hline 8 & 0.9968 & 0.9874 & 0.9856 & 0.9839 & 0.0010 & 0.0003 & 0.0001 & 0.0001 & 0.0001 \\
\hline 9 & 0.9961 & 0.9855 & 0.9838 & 0.9808 & 0.0007 & 0.0002 & 0.0001 & 0.0001 & 0.00001 \\
\hline 10 & 0.9951 & 0.9831 & 0.9823 & 0.9713 & 0.0007 & 0.0002 & 0.0001 & 0.0001 & 0.00001 \\
\hline
\end{tabular}


As we can see from table -3 , the percentile points of $\mathrm{T}_{2}$ increase as the smple size increases as well as the significance level increases. The power of the test statistic $\mathrm{T}_{1}$ is also tabulate for 3 different levels of significance $(1 \%, 2.5 \%, 5 \%)$ at the sample sizes $n=2,3 \ldots 10$. by simulating sampling from $\mathrm{P}_{1}$ and using the values of $\mathrm{T}_{2}$. The count of $\mathrm{T}_{2}$ values that fall beyond the table values of table -3 . These are given in Table-4.

Table-4 Power of $\mathbf{T}_{\mathbf{2}}$

\begin{tabular}{|c|c|c|c|}
\hline $\mathbf{n}$ & $\mathbf{0 . 9 9}$ & $\mathbf{0 . 9 7 5}$ & $\mathbf{0 . 9 5}$ \\
\hline 2 & 9901 & 9754 & 9500 \\
\hline 3 & 9978 & 9877 & 9699 \\
\hline 4 & 9980 & 9892 & 9774 \\
\hline 5 & 9985 & 9897 & 9781 \\
\hline 6 & 9972 & 9869 & 9723 \\
\hline 7 & 9990 & 9926 & 9799 \\
\hline 8 & 9987 & 9897 & 9742 \\
\hline 9 & 9985 & 9902 & 9754 \\
\hline 10 & 9991 & 9958 & 9938 \\
\hline
\end{tabular}

\section{COMPARATIVE STUDY\&CONCLUSION}

The powers given in table $2 \& 4$ for the test statistics $\mathrm{T}_{1}$ and $T_{2}$ respectively indicate that the two statistics do not discriminate between Dagum \& Burr-III distributions significantly. i.e there is much similarity between these two distributions for larger values of $\mathrm{n}$. If the sample size increases the Dagum distribution and Burr-3 distribution cannot be distinguished with respect to proposed test statistics. We may say that for a data from Dagum distribution and the application of Dagum distribution is quite complicated, the methodology of Burr-III distribution can be used in large samples.

\section{ACKNOWLEDGMENT}

The authors thank the editor and the reviewer for their helpful suggestions, comments and encouragement, which helped in improving the final version of the paper.

\section{REFERENCES}

[1] Arabin.K.D. and Kundu.D., "Discriminating between the $\log$ normal and log logistic distributions",Communications in Statistics Theory and Methods, Vol 39, pp.280-290, 2010.

[2] Chambers.E.A. and Cox.D.R "Discriminating Between alternative binary response models", Biometrika, Vol.54, pp 573-578, 1967.

[3] Dumonceaux.R. and Antle.C. E., "Discriminating between the lognormal and weibull distributions", Technometrics, Vol.15, pp 923-926, 1973

[4] Gupta.D.R., Kundu.D., "Discriminating between gamma and generalized exponential distributions" , Journal of Statistical computation and Simulation, Vol.74,No.2,pp 107-121,2004.
[5] Gupta.D.R., Kundu.D.,Manglic.A., Discriminating between log normal and generalized exponential distributions, Journal of Statistical planning and Inference, Vol.127: pp 213-227, 2005.

[6] Rao.B.S. and Kantam.R.R.L., "Discriminating between generalized exponential distribution and some life test models based on population quantiles", Journal of Modern Applied StatisticalMethods, Vol.12 No.2, pp.336-343, 2013.

[7] Rao.B.S. and Kantam.R.R.L., "Discriminating between loglogistic and Rayleigh distributions", Pakistan Journal of Statistics and Operations Research, Vol. 10,No.1, pp. 1-7, 2014.

\section{AUTHORS PROFILE}

B.Srinivasa Rao did his M.Sc in Statistics from Acharya Nagarjuna University in 1996. He completed his doctoral research in the area of Software Reliability and Quality control methods from Acharya Nagarjuna University in 2010. Currently he is working as an Associate Professor of Statistics at R.V.R \& J.C College of Engineering, Guntur

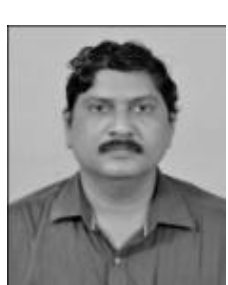
,putting an experience of 22 years in teaching. He published more than 35 research articles in reputed national and international Journals. His areas of specialization are Reliability, Quality control methods and Statistical inference. His teaching interests include Bio-Statistics, Quantitative techniques, Probability, Inferential Statistics and Stochastic process.

P.Sricharani is currently working as Assistant Professor in department of Basic science from Shri Vishnu Engineering college for women, Bhimavaram. She guided for 4 P.G Projects. she has 13 years of teaching experience. Her research interest is in the area of Statistical Quality Control and Statistical Inference. She has published many research papers in National and International journals. 